\title{
Designing Futuristic Telemedicine Using Artificial Intelligence and Robotics in the COVID-19 Era
}

\begin{abstract}
Sonu Bhaskar ${ }^{1,2 *}$, Sian Bradley ${ }^{1,3}$, Sateesh Sakhamuri ${ }^{1,4}$, Sebastian Moguilner ${ }^{1,5}$, Vijay Kumar Chattu 1,6, Shawna Pandya ${ }^{1,7}$, Starr Schroeder ${ }^{1,8}$, Daniel Ray ${ }^{1,9}$ and Maciej Banach ${ }^{1,10,11}$

${ }^{1}$ Pandemic Health System REsilience PROGRAM (REPROGRAM) Consortium, REPROGRAM Telemedicine Study Group, Sydney, NSW, Australia, ${ }^{2}$ Neurovascular Imaging Laboratory \& NSW Brain Clot Bank, Department of Neurology, Liverpool Hospital and South Western Sydney Local Health District, Ingham Institute for Applied Medical Research, The University of New South Wales, Sydney, NSW, Australia, ${ }^{3}$ The University of New South Wales (UNSW) Medicine Sydney, South West Sydney Clinical School, Sydney, NSW, Australia, ${ }^{4}$ The University of the West Indies, St. Augustine, Trinidad and Tobago, ${ }^{5}$ Global Brain Health Institute, Trinity College Dublin, Dublin, Ireland, ${ }^{6}$ Department of Medicine, St. Michael's Hospital, University of Toronto, Toronto, ON, Canada, ${ }^{7}$ Alberta Health Services and Project PoSSUM, University of Alberta, Edmonton, AB, Canada, ${ }^{8}$ Penn Medicine Lancaster General Hospital and Project PoSSUM, Lancaster, PA, United States, ${ }^{9}$ Farr Institute of Health Informatics, University College London (UCL) \& NHS Foundation Trust, Birmingham, United Kingdom, ${ }^{10}$ Polish Mother's Memorial Hospital Research Institute (PMMHRI) in Lodz, Cardiovascular Research Centre, University of Zielona Gora, Zielona Gora, Poland, ${ }^{11}$ Department of Hypertension, Medical University of Lodz, Łódź, Poland
\end{abstract}

OPEN ACCESS

Edited by:

Alberto Eugenio Tozzi,

Bambino Gesù Children Hospital (IRCCS), Italy

Reviewed by:

Rukhsana Ahmed,

University at Albany, United States

Charles Doarn,

University of Cincinnati, United States

*Correspondence:

Sonu Bhaskar

Sonu.Bhaskar@health.nsw.gov.au

Specialty section:

This article was submitted to

Digital Public Health,

a section of the journal

Frontiers in Public Health

Received: 30 April 2020

Accepted: 07 October 2020

Published: 02 November 2020

Citation:

Bhaskar S, Bradley S, Sakhamuri S, Moguilner S, Chattu VK, Pandya S, Schroeder S, Ray D and Banach M

(2020) Designing Futuristic

Telemedicine Using Artificial

Intelligence and Robotics in the

COVID-19 Era.

Front. Public Health 8:556789.

doi: 10.3389/fpubh.2020.556789
Technological innovations such as artificial intelligence and robotics may be of potential use in telemedicine and in building capacity to respond to future pandemics beyond the current COVID-19 era. Our international consortium of interdisciplinary experts in clinical medicine, health policy, and telemedicine have identified gaps in uptake and implementation of telemedicine or telehealth across geographics and medical specialties. This paper discusses various artificial intelligence and robotics-assisted telemedicine or telehealth applications during COVID-19 and presents an alternative artificial intelligence assisted telemedicine framework to accelerate the rapid deployment of telemedicine and improve access to quality and cost-effective healthcare. We postulate that the artificial intelligence assisted telemedicine framework would be indispensable in creating futuristic and resilient health systems that can support communities amidst pandemics.

Keywords: telehealth, digital medicine, pandemic (COVID-19), robotics, telemedicine, artificial intelligence, coronavirus disease 2019 (COVID-19)

\section{INTRODUCTION}

Telemedicine or telehealth is the use of medical information to improve patient's health $(1,2)$. Reorganization in healthcare delivery, financing, and advancement in electronic health records and clinical decision support systems can accelerate the telehealth adoption into healthcare delivery (2). In this context, artificial intelligence (AI) and robotic technologies can play an important role in the use and delivery of telemedicine during coronavirus disease (COVID-19) and the post-pandemic world (3-10). Our previous two reports have identified gaps in telemedicine across geographics and medical specialties $(11,12)$. The current paper is a call for the integration of AI, robotics, and telemedicine with an organizational framework powered by AI to accelerate healthcare delivery and improve access to healthcare in the context of public health preparedness and response during outbreaks or public health emergencies such as COVID-19. 


\section{ARTIFICIAL INTELLIGENCE ASSISTED TELEMEDICINE}

Diagnosis is a multidisciplinary process that may involve multimodal testing such as clinical, imaging, blood, and genetic markers. Moreover, discipline-specific testing such as neuropsychological tests may be needed, for example, to obtain comprehensive mental health assessments (13). In a telemedicine framework, some of these testing may be unavailable, while others may be cost-prohibitive. To address this complex multivariate problem in finding an optimal diagnostic protocol, innovative data-driven artificial intelligence (AI) algorithms may offer a solution by applying machine learning to large datasets of disease populations $(14,15)$. These models can learn directly from the data without any prior statistical modeling, thus producing more objective results while focusing on prediction generalizability for diagnostic purposes on diverse populations. Since the COVID-19 outbreak, international efforts toward COVID-19 forecasting, prevention and treatment are underway using data-driven tools and pooled datasets (16). Moreover, the ML model features an important analysis that enables the search for more cost-effective protocols (17). Unlike traditional statistical hypothesis testing, data-driven computational approaches can test for synergistic variable combinations and redundant feature elimination enabling more effective diagnosis under the specific constraints of telemedicine (18).

Analysis by Collier et al. found that the use of AI applications could result in $\sim \$ 150$ billion in saved healthcare costs annually by 2026 in the United States (19). According to Wahl et al., the ubiquitous use of smartphones, combined with growing investments in supporting technologies (e.g., mHealth, electronic medical record (EMR), and cloud computing), provide ample opportunities to use AI applications to improve public health outcomes in low-income country settings (20). Rapidly increased usage of electronic gadgets accelerates digital shifts in healthcare that appear to become essential in sharing information between and within medical workers and patients (21).

AI exhibits clear advantages over humans in analytical reasoning and problem solving (especially when large amounts of data are involved) and can effectively address the limitations of human function (22). However, the use of AI in healthcare must consider or mitigate the potential loss of vital physician skills if AI is over-utilized (17). Furthermore, rigid algorithm protocols and decision-making trees are subject to the consequences of the inability of AI to fully take in and interpret contextual information or delineate between relevant vs. non-relevant informational input even when employing deep machine learning (23). Contingencies are the norm in healthcare, and the human skill required to navigate and manage this offnominal, or unpredictable situations must be carefully weighed against the advantages of using AI technology (23-28). User interface and data input methods are critical as voice recognition and interpretation is a major challenge of AI utilization (29). Generalized challenges to utilizing AI in healthcare are created by the fact that many cares and treatment decisions, especially in emergent and time-restricted scenarios hinge on human thought processes that occur in the subconscious, such as intuition, insight, subjective evaluation, and the analyzation of ambiguous or qualitative data (30). Jarrahi illustrates the benefit of the symbiotic use of AI combined with a human in the loop by showing the statistically significant reduction of error (85\%) in detecting cancer in images of lymph nodes when compared to AI or human interpretation alone (30).

Telemedicine has provided a critical patient continuity pathway in times of disruption in health service during COVID19 (10-12). This also helps protect healthcare facilities and minimize risks to health workers during pandemics, which/who are increasingly under pressure (31-33). Telemedicine has the potential to minimize the economic impact on society and healthcare services. Quarantined doctors can provide these services in medical institutions with remote access or care via telecommunications directly to the consumer, freeing other doctors to provide immediate assistance to more needy patients. Teleconsultations allow doctors to evaluate patients, detect signs of infection, and quickly and easily document patients who might be at higher risk of illness (34). Telemedicine solutions work promptly, filling out insurance documents, which allows doctors to devote more time to treating patients. Clinics are expanding their telehealth services to screen patients for COVID-19, which is critical to identifying patients and speeding up medical care, as well as limiting public exposure (11, 32). Bundling of various specialties such as teleradiology, tele-oncology, and telepathology is another area of crucial consideration to facilitate comprehensive management (35).

Further upstream in the telemedical framework, it is important to consider the integration of educational models for physicians and trainees, and particularly how these technologies may address the issues of connecting distributed learners (particularly in the era of a pandemic) (36), and providing highquality educating, training, and just-in-time training, in a way that is trusted, safe, and replicates the quality of "in-person" training, particularly when it comes to surgical procedures $(37,38)$. Immersive technologies such as virtual reality and augmented reality could enhance capabilities for collaboration, both amongst learners and physicians in practice (39). Lastly, as has been mentioned elsewhere in this paper, immersive and telemedical technologies, whether used for consultations, treatments, and diagnostics, or education, must be resilient and capable of maintaining at least some degree of functionality even in the absence of internet or network connectivity (40). This is relevant to areas where broadband internet access is poor which limits the effective implementation of these technologies (41).

\section{ROBOTICS ASSISTED TELEMEDICINE}

Robotics is a promising cutting-edge tool in telemedicine that has potential applications in transforming physical exam and clinical care, as well as monitoring patients in remote conditions $(42,43)$. Such experience with the Ebola outbreak and with the COVID-19 pandemic has revealed a wide range of robotic uses and telemedicine options $(43,44)$. Robotics can be used in outbreaks of infections to minimize further exposure $(45,46)$ : 


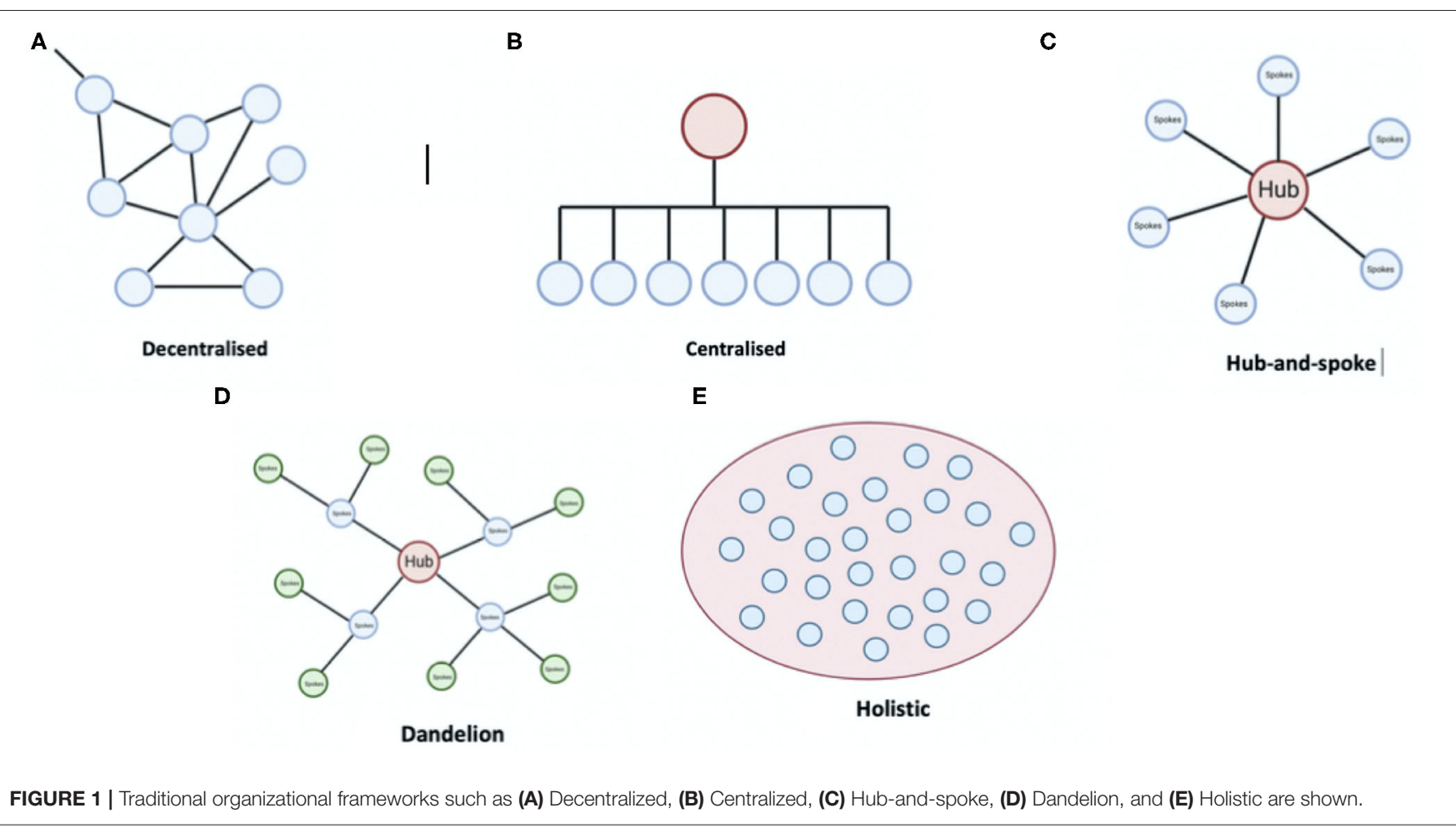

for disinfection, delivery of drugs and food, measuring vital signs, facilitating border control, and automatic disinfection (43). Telepresence robots allow for two-way communication and can be remotely controlled to provide support to those in isolation by connecting patients with family and physicians (35). Exposure to COVID-19 may stimulate further robotics research to address the risk of infectious diseases (42). Facilitating the integration of engineering, video technology, and infectious diseases specialists with government funding can have a notable impact on preventing future pandemics. A Smart Field Hospital trial in Wuhan, China, used robots to minimize COVID-19 exposure to patients and healthcare workers as robots and internet of things (IoT) devices provided medical services in the facility (47). The adoption of robots could be explored in infectious disease and crisis settings as a means to potentially improve health systems capacity and preparedness (47).

The Society of European Robotic Gynecological Surgery has released guidelines for robot-assisted surgery (RAS) and promoted the use of RAS over conventional laparoscopic and open surgery in managing infection risk (48). Where RAS is not possible, conventional laparoscopic is preferred over open surgery due to the reduced amount of physicians and PPE required, aerosol and bodily fluid risk, and shortened hospital stay $(48,49)$. The European Association of Urology similarly released COVID-19 guidelines, in which they pressed the need to manage smoke dispersion in robotic or laparoscopic surgery through the use of lowest intra-abdominal pressure and management of flow systems (50).

Potential issues associated with robotics-assisted telemedicine, include precision and interaction issues due to distance between patient and operator, network issues, and communication issues (49). Robot-assisted surgery has the potential to reduce COVID19 exposure risk to medical professionals (51).

\section{TELEMEDICINE ORGANIZATIONAL FRAMEWORKS}

Various traditional organizational frameworks are illustrated in Figure 1. One example of a traditional organization is the huband-spoke framework for stroke reperfusion therapy delivery (52), which is based on a framework of conventional and hierarchical positioning. It involves a centralized hub, which serves as a point of contact and instruction to several spoke sites that deliver care (53). This structure is suitable for the set-up and integration of telehealth networks but ultimately slows decisionmaking, depresses innovation, makes it difficult for spokes to communicate, and not everybody in the spoke has the capability of the hub (54). Due to cost-benefit and infrastructural reasons, as well as a shortage of neuro-interventionalists, not every spoke will have endovascular therapy capabilities. Additionally, barriers in access to health systems, applicable to culturally and linguistically diverse communities (CALD) as well as those from marginalized backgrounds or low resourced settings, merit special consideration $(55,56)$.

Centralized systems require strong leadership, otherwise, issues such as lack of efficiency, productivity, and physician wellbeing may develop (57). Decentralized systems can slow down the speed of uptake and pose inefficiency. Multiple hub-andspokes are another framework that can be leveraged (58), e.g., 


\section{Proposed Al-powered telemedicine framework}

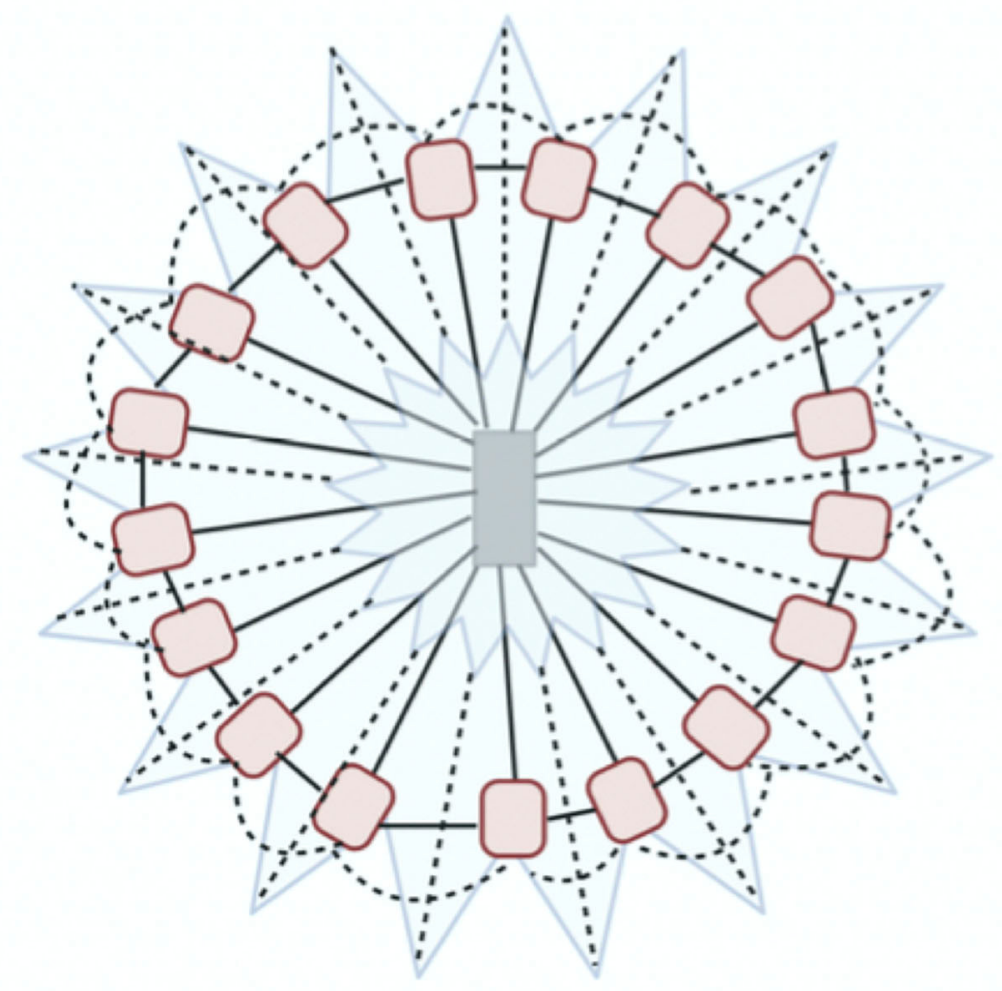

FIGURE 2 | The proposed artificial intelligence assisted framework for telemedicine. This system centers around artificial intelligence (Al) engine that looks within the network to find the optimal resources within the geographical constraints and route incoming calls to the appropriate node (affiliated healthcare provider/facility). This self-evolving and innovative approach processing system would potentially allow increased telemedicine penetration while reducing the inefficiencies of the top-down or conventional organizational frameworks.

in Africa, a capital city can have a hub overseeing the local state governments, which could further branch out. There also exists a holistic model, in which there is no hierarchy (59). Each element in the holistic structure is guided by the overarching vision of the organization.

\section{DISCUSSION}

Telemedicine offers a new modality of delivering medical \& allied health services and communicating with patients (11). The current COVID-19 pandemic has catalyzed the uptake of telemedicine $(12,60)$. However, there are challenges concerning its geographical penetration, organizational structures, and infrastructure-related issues $(12,41)$. The consortium has outlined gaps in current telehealth approaches (11), including challenges to telemedicine implementation across geographics (12) and reliance on outdated organizational frameworks or operational structures discussed in the current report.

We propose a novel AI-powered staged-wise telemedicine organizational framework (61), which can potentially overcome the challenges associated with traditional organizational structures (Figure 2). Hub-and-spoke can be used as a foundational set-up to trigger the quick adoption of technology initially. However, after an initial period, once such a structure begins to stagnate and is no longer effective in skilling-up, a new organizational framework will replace the foundational model. The replacing model will consider leadership, technological, and organizational structure competence (61). This will involve a move toward performance and competency-based systems (62). For example, when an acute stroke call is made, an AI system will automatically find the best site of care depending on several variables, distance, availability of resources, clinicians' availability, and time constraints. This will focus on linking the patient to the best provider, thereby removing any elements of bureaucracy. An independent governance and ethical framework are necessary for oversight over the AI performance and any ethical issues $(63,64)$. An AI-powered system should prioritize the collective good and performance of the organization based on evidence-based management principles (65). It will be utilized to form linkages between providers. For instance, in the event of a cardiac emergency, the system could use the existing information on the patient to find the best teams to manage the patient. 
Links between a cardiologist and cardiac surgeon will be made so that they can communicate quickly. This involves role-based linkage based on questions such as who is available quickly, who is available in the region, and whether the practitioner agrees to treat such patients, rather than subjective person-to-person linkage. Ultimately, however, such an autonomous framework must also be able to assign responsibility in case of oversights. Hub-and-spoke systems allow teams to compete with each other and to curry favor from management. The proposed AI-assisted telemedicine organizational framework is focused on role-competence and linkages, rather than individuals or teams. We anticipate this would foster regular and continuous collaboration. Powered by big data and advanced data analytics dashboard, AI-powered systems can be insightful because they generate information about resource utilization in different regions, providing recommendations on system reorganization and clinician mobilization in a COVID-19 pandemic situation. This involves scoping the landscape and realigning across multiple levels to embed that particular intervention within the health systems, thereby promoting rapid research and innovation translation as well as integrating contributions based on local needs. Such a framework would imbibe resilience, innovationdriven technology, and scaled intelligence to enable systems to evolve and be responsive to local and emergent needs such as during an outbreak. COVID-19 has led to a sharp increase in the demand for telemedicine services $(5,34)$. It also has an impact on telemedicine providers, a sector that is facing unprecedented demand, which by some estimates has grown by $150 \%$ or more (66). For telemedicine to expand across geographics, it is necessary to account for geographical variations, cultural factors, and involvement of local stakeholders (12).

\section{CONCLUSION}

To summarize, AI and robotics could play an important role in providing telemedicine services during an outbreak or public health emergency while limiting exposure to healthcare workers and health systems (14, 42, 43). The AI-assisted

\section{REFERENCES}

1. World Health Organization. Transforming and Scaling up Health Professionals' Education and Training. Geneva: World Health Organization (2013). Available online at: https://www.who.int/hrh/resources/transf_ scaling_hpet/en/ (accessed September 14, 2020).

2. Tuckson RV, Edmunds M, Hodgkins ML. Telehealth. N Engl J Med. (2017) 377:1585-92. doi: 10.1056/NEJMsr1503323

3. Ye J. The role of health technology and informatics in a global public health emergency: practices and implications from the COVID-19 pandemic. JMIR Med Inform. (2020) 8:e19866. doi: 10.2196/19866

4. Agbehadji IE, Awuzie BO, Ngowi AB, Millham RC. Review of big data analytics, artificial intelligence and nature-inspired computing models towards accurate detection of COVID-19 pandemic cases and contact tracing. Int J Environ Res Public Health. (2020) 17:5330. doi: 10.3390/ijerph17 155330

5. Ohannessian R, Duong TA, Odone A. Global telemedicine implementation and integration within health systems to fight the COVID-19 pandemic: a call to action. JMIR Public Health Surveill. (2020) 6:e18810. doi: 10.2196/18810 telemedicine framework proposed by the current consortium could be an enabler in improving telemedicine access and spread across medical specialties and geography. An international collaborative effort led by WHO, the current consortium, or similar organizations could pave a way to greater telemedicine penetration, especially to benefit the underprivileged and those living in the low-resourced settings (12).

\section{AUTHOR'S NOTE}

The COVID-19 pandemic is causing an unprecedented public health crisis impacting healthcare systems, healthcare workers, and communities. The COVID-19 Pandemic Health System REsilience PROGRAM (REPROGRAM) consortium is formed to champion the safety of healthcare workers, policy development, and advocacy for global pandemic preparedness and action.

\section{AUTHOR CONTRIBUTIONS}

SBh devised the project, the main conceptual ideas, including the proposal for a new AI-powered telemedicine workflow, the proof outline, coordinated the writing, editing of the manuscript, and wrote the first draft of the manuscript. All authors discussed the results and recommendations and contributed to the final manuscript.

\section{ACKNOWLEDGMENTS}

We would like to acknowledge the REPROGRAM consortium members who have worked tirelessly over the last days in contributing to various guidelines, recommendations, policy briefs, and ongoing discussions during these unprecedented and challenging times despite the incredibly short timeframe. We would like to dedicate this work to our healthcare workers who have died due to COVID-19 while serving the patients at the frontline and to those who continue to serve during these challenging times despite lack of personal protective equipment.

6. Mahmood S, Hasan K, Colder Carras M, Labrique A. Global preparedness against COVID-19: we must leverage the power of digital health. JMIR Public Health Surveill. (2020) 6:e18980. doi: 10.2196/18980

7. Uohara MY, Weinstein JN, Rhew DC. The essential role of technology in the public health battle against COVID-19. Popul Health Manag. (2020) 23:361-7. doi: 10.1089/pop.2020.0187

8. Badawy SM, Radovic A. Digital approaches to remote pediatric health care delivery during the COVID-19 pandemic: existing evidence and a call for further research. JMIR Pediatr Parent. (2020) 3:e20049. doi: 10.2196/20049

9. Kricka LJ, Polevikov S, Park JY, Fortina P, Bernardini S, Satchkov D, et al. Artificial intelligence-powered search tools and resources in the fight against COVID-19. EJIFCC. (2020) 31:106-16.

10. Hollander JE, Carr BG. Virtually perfect? telemedicine for Covid-19. N Engl J Med. (2020) 382:1679-81. doi: 10.1056/NEJMp2003539

11. Bhaskar S, Bradley S, Chattu VK, Adisesh A, Nurtazina A, Kyrykbayeva S, et al. Telemedicine as the new outpatient clinic gone digital: position paper from the pandemic health system REsilience PROGRAM (REPROGRAM) international consortium (Part 2). Front Public Health. (2020) 8:410. doi: $10.3389 /$ fpubh. 2020.00410 
12. Bhaskar S, Bradley S, Chattu VK, Adisesh A, Nurtazina A, Kyrykbayeva $\mathrm{S}$, et al. Telemedicine across the globe - position paper from the COVID-19 pandemic health system resilience PROGRAM (REPROGRAM) international consortium (Part 1). Front Public Health. (2020) 8:556720. doi: 10.3389/fpubh.2020.556720

13. Huys QJM, Maia TV, Frank MJ. Computational psychiatry as a bridge from neuroscience to clinical applications. Nat Neurosci. (2016) 19:404-13. doi: $10.1038 / \mathrm{nn} .4238$

14. Kuziemsky C, Maeder AJ, John O, Gogia SB, Basu A, Meher S, et al. Role of artificial intelligence within the telehealth domain. Yearb Med Inform. (2019) 28:35-40. doi: 10.1055/s-0039-1677897

15. Aractingi S, Pellacani G. Computational neural network in melanocytic lesions diagnosis: artificial intelligence to improve diagnosis in dermatology? Eur J Dermatol. (2019) 29:4-7. doi: 10.1684/ejd.2019.3538

16. Luo EM, Newman S, Amat M, Charpignon ML, Duralde ER, Jain S, et al. MIT COVID-19 datathon: data without boundaries. BMJ Innovations. (2020). doi: 10.1136/bmjinnov-2020-000492. [Epub ahead of print].

17. Ahuja AS. The impact of artificial intelligence in medicine on the future role of the physician. PeerJ. (2019) 7:e7702. doi: 10.7717/peerj.7702

18. Pacis DMM, Subido EDC, Bugtai NT. Trends in telemedicine utilizing artificial intelligence. AIP Conf Proc. (2018) 1933:040009. doi: $10.1063 / 1.5023979$

19. Accenture. Why Artificial Intelligence Is the Future of Growth. (2020). Available online at: https:/www.accenture.com/us-en/insight-artificialintelligence-future-growth (accessed September 19, 2020).

20. Wahl B, Cossy-Gantner A, Germann S, Schwalbe NR. Artificial intelligence (AI) and global health: how can AI contribute to health in resource-poor settings? BMJ Global Health. (2018) 3:e000798. doi: 10.1136/bmjgh-2018-000798

21. Thierry JP. [The human resource: cornerstone of the digital transformation of the healthcare plan]. Soins. (2020) 65:46-7. doi: 10.1016/j.soin.2020.01.009

22. Huang M-H, Rust RT. Artificial intelligence in service. J Serv Res. (2018) 21:155-72. doi: 10.1177/1094670517752459

23. Heinrichs B, Eickhoff SB. Your evidence? Machine learning algorithms for medical diagnosis and prediction. Hum Brain Mapp. (2020) 41:1435-44. doi: $10.1002 / \mathrm{hbm} .24886$

24. Grote T, Berens P. On the ethics of algorithmic decision-making in healthcare. J Med Ethics. (2020) 46:205-11. doi: 10.1136/medethics-2019-105586

25. London AJ. Artificial intelligence and black-box medical decisions: accuracy versus explainability. Hastings Cent Rep. (2019) 49:15-21. doi: $10.1002 /$ hast.973

26. McDougall RJ. Computer knows best? The need for valueflexibility in medical AI. J Med Ethics. (2019) 45:156-60. doi: 10.1136/medethics-2018-105118

27. Sun W, Nasraoui O, Shafto P. Evolution and impact of bias in human and machine learning algorithm interaction. PLoS ONE. (2020) 15:e0235502. doi: 10.1371/journal.pone.0235502

28. Ash JS, Berg M, Coiera E. Some unintended consequences of information technology in health care: the nature of patient care information system-related errors. J Am Med Inform Assoc. (2004) 11:104-12. doi: 10.1197/jamia.M1471

29. Davenport T, Kalakota R. The potential for artificial intelligence in healthcare. Future Healthc J. (2019) 6:94-8. doi: 10.7861/futurehosp.6-2-94

30. Jarrahi $\mathrm{MH}$. Artificial intelligence and the future of work: human-AI symbiosis in organizational decision making. Bus Horiz. (2018) 61:577-86. doi: 10.1016/j.bushor.2018.03.007

31. Bhaskar S, Bradley S, Israeli-Korn S, Menon B, Chattu VK, Thomas P, et al. Chronic neurology in COVID-19 era: clinical considerations and recommendations from the REPROGRAM consortium. Front Neurol. (2020) 11:664. doi: 10.3389/fneur.2020.00664

32. Bhaskar S, Rastogi A, Chattu VK, Adisesh A, Thomas P, Alvarado N, et al. Key strategies for clinical management and improvement of healthcare services for cardiovascular disease and diabetes patients in the coronavirus (COVID19) settings: recommendations from the REPROGRAM consortium. Front Cardiovasc Med. (2020) 7:112. doi: 10.3389/fcvm.2020.00112

33. Bhaskar S, Sharma D, Walker AH, McDonald M, Huasen B, Haridas A, et al. Acute neurological care in the COVID-19 era: the pandemic health system
REsilience PROGRAM (REPROGRAM) consortium pathway. Front Neurol. (2020) 11:579. doi: 10.3389/fneur.2020.00579

34. Sinha S, Kern LM, Gingras LF, Reshetnyak E, Tung J, Pelzman F, et al. implementation of video visits during COVID-19: lessons learned from a primary care practice in New York City. Front Public Health. (2020) 8:514. doi: 10.3389/fpubh.2020.00514

35. Bakas T, Sampsel D, Israel J, Chamnikar A, Ellard A, Clark JG, et al. Satisfaction and technology evaluation of a telehealth robotic program to optimize healthy independent living for older adults. J Nurs Scholarsh. (2018) 50:666-75. doi: 10.1111/jnu.12436

36. Lucey CR, Johnston SC. The transformational effects of COVID-19 on medical education. JAMA. (2020) 324:1033-4. doi: 10.1001/jama.2020.14136

37. Jumreornvong $O$, Yang E, Race J, Appel J. Telemedicine and medical education in the age of COVID-19. Acad Med. (2020). doi: 10.1097/ACM.0000000000003711. [Epub ahead of print].

38. Aron JA, Bulteel AJB, Clayman KA, Cornett JA, Filtz K, Heneghan L, et al. A role for telemedicine in medical education during the COVID-19 pandemic. Acad Med. (2020). doi: 10.1097/ACM.0000000000003572. [Epub ahead of print].

39. Bremner R, Gibbs A, Mitchell ARJ. The era of immersive health technology. EMJ Innov. (2020) 4:40-7.

40. Institute of Medicine (US) Committee on Assuring the Health of the Public in the 21st Century. The Future of the Public's Health in the 21st Century. Washington, DC: National Academies Press (2002). The Health Care Delivery System. Available online at: https://www.ncbi.nlm.nih.gov/books/ NBK221227/ (accessed September 19, 2020).

41. Drake C, Zhang Y, Chaiyachati KH, Polsky D. The limitations of poor broadband internet access for telemedicine use in Rural America: an observational study. Ann Intern Med. (2019) 171:382-4. doi: 10.7326/M19-0283

42. Tavakoli M, Carriere J, Torabi A. Robotics, smart wearable technologies, and autonomous intelligent systems for healthcare during the COVID-19 pandemic: an analysis of the state of the art and future vision. Adv Intell Syst. (2020) 2:2000071. doi: 10.1002/aisy.202000071

43. Yang G-Z, Nelson JB, Murphy RR, Choset H, Christensen H, Collins $\mathrm{HS}$, et al. Combating COVID-19-The role of robotics in managing public health and infectious diseases. Sci Robot. (2020) 5:eabb5589. doi: 10.1126/scirobotics.abb5589

44. Deng B. Robo-rescuers battle it out in disaster challenge. Nature. (2015) 519:14-5. doi: 10.1038/519014a

45. Kovach CR, Taneli Y, Neiman T, Dyer EM, Arzaga AJA, Kelber ST. Evaluation of an ultraviolet room disinfection protocol to decrease nursing home microbial burden, infection and hospitalization rates. BMC Infect Dis. (2017) 17:186. doi: 10.1186/s12879-017-2275-2

46. Yang G, Lv H, Zhang Z, Yang L, Deng J, You S, et al. Keep healthcare workers safe: application of teleoperated robot in isolation ward for COVID-19 prevention and control. Chin J Mech Eng. (2020) 33:47. doi: 10.1186/s10033-020-00464-0

47. Hornyak T. CNBC. What America can learn from China's use of robots and telemedicine to combat the coronavirus. (2020). Available online at: https:// www.cnbc.com/2020/03/18/how-china-is- using-robots-and-telemedicineto-combat-the-coronavirus.html (accessed September 14, 2020).

48. Kimmig R, Verheijen RHM, Rudnicki M. Robot assisted surgery during the COVID-19 pandemic, especially for gynecological cancer: a statement of the Society of European Robotic Gynaecological Surgery (SERGS). J Gynecol Oncol. (2020) 31:e59. doi: 10.3802/jgo.2020.31.e59

49. Avgousti S, Christoforou EG, Panayides AS, Voskarides S, Novales C, Nouaille L, et al. Medical telerobotic systems: current status and future trends. BioMed Eng Online. (2016) 15:96. doi: 10.1186/s12938-016-0217-7

50. Mottrie A. ERUS 2020. ERUS (EAU Robotic Urology Section) guidelines during COVID-19 emergency. (2020). Available online at: https://uroweb. org/wp-content/uploads/ERUS-guidelines-for-COVID-def.pdf (accessed 27 April 2020).

51. Samalavicius NE, Siaulys R, Janusonis V, Klimasauskiene V, Dulskas A. Use of 4 robotic arms performing Senhance $\AA$ robotic surgery may reduce the risk of coronavirus infection to medical professionals during COVID-19. Eur J Obstet Gynecol Reprod Biol. (2020) 251:274-5. doi: 10.1016/j.ejogrb.2020.06.014 
52. Demaerschalk BM, Boyd EL, Barrett KM, Gamble DM, Sonchik S, Comer MM, et al. Comparison of stroke outcomes of hub and spoke hospital treated patients in mayo clinic telestroke program. J Stroke Cerebrovasc Dis. (2018) 27:2940-2. doi: 10.1016/j.jstrokecerebrovasdis.2018.06.024

53. Subramanian S, Pamplin JC, Hravnak M, Hielsberg C, Riker R, Rincon $\mathrm{F}$, et al. Tele-critical care: an update from the society of critical care medicine tele-ICU committee. Crit Care Med. (2020) 48:553-61. doi: 10.1097/CCM.0000000000004190

54. Elrod JK, Fortenberry JL. The hub-and-spoke organization design: an avenue for serving patients well. BMC Health Serv Res. (2017) 17:457. doi: 10.1186/s12913-017-2341-x

55. Bhaskar S, Thomas P, Cheng Q, Clement N, McDougall A, Hodgkinson $S$, et al. Trends in acute stroke presentations to an emergency department: implications for specific communities in accessing acute stroke care services. Postgraduate Med J. (2019) 95:258-64. doi: 10.1136/postgradmedj-2019-136413

56. Santana Baskar P, Cordato D, Wardman D, Bhaskar S. In-hospital acute stroke workflow in acute stroke - systems-based approaches. Acta Neurol Scand. (2020). doi: 10.1111/ane.13343. [Epub ahead of print].

57. Vantrappen H, Wirtz F. Harvard Business Review. When to Decentralize Decision Making, and When Not To. (2020). Available online at: https:// hbr.org/2017/12/when-to-decentralize-decision-making-and-when-not-to (accessed September 14, 2020).

58. de Camargo RS, Miranda Jr G, Ferreira RPM, Luna HP. Multiple allocation hub-and-spoke network design under hub congestion. Comp Operat Res. (2009) 36:3097-106. doi: 10.1016/j.cor.2008.10.004

59. Doherty N, Champion D, Wang L. An holistic approach to understanding the changing nature of organisational structure. IT People. (2010) 23:116-35. doi: 10.1108/09593841011052138

60. Fisk M, Livingstone A, Pit SW. Telehealth in the context of COVID19: changing perspectives in Australia, the United Kingdom, and the United States. J Med Internet Res. (2020) 22:e19264. doi: 10.2196/19264

61. Fountaine T, McCarthy B, Saleh T. Harvard Business Review. Building the AIPowered Organization. (2019). Available online at: https://hbr.org/2019/07/ building-the-ai-powered-organization (accessed September 19, 2020).

62. Korenková V, Závadský J, Lis M. Linking a performance management system and competencies: qualitative research.
Eng Manag Prod Serv. (2019) 11:51-67. doi: 10.2478/emj2019-0004

63. Anand M. Knowledge@Wharton. Want Responsible AI? Think Business Outcomes" Knowledge@Wharton. (2020). Available online at: https:// knowledge.wharton.upenn.edu/article/want-responsible-ai-think-businessoutcomes/ (accessed September 13, 2020).

64. Cobey C, Boillet J. EY. How do You Teach AI the Value of Trust? (2020). Available online at: https://www.ey.com/en_gl/ digital/how-do-you-teach-ai-the-value-of-trust (accessed September 14, 2020).

65. West DM, Allen JR. Brookings. How artificial intelligence is transforming the world. (2018). Available online at: https://www.brookings.edu/research/ how-artificial-intelligence-is-transforming-the-world/ (accessed September 14, 2020).

66. Frost \& Sullivan 2020. Telehealth-A Technology-Based Weapon in the War against the Coronavirus. Social Distancing Needs will Present Both Growth Opportunities and Challenges. (2020). Available online at: https:// go.frost.com/NA_PR_TH_MFernandez_K488_Telehealth_May20 (accessed September 19, 2020).

Conflict of Interest: SP is the Vice President of Immersive Medicine at Luxsonic technologies, a medical technology company specializing in virtual/augmented reality for medical education, collaboration, and training. The opinions expressed in this article are those of the authors and do not necessarily represent the decisions, official policy, or opinions of the affiliated institutions.

The remaining authors declare that the research was conducted in the absence of any commercial or financial relationships that could be construed as a potential conflict of interest.

Copyright (C) 2020 Bhaskar, Bradley, Sakhamuri, Moguilner, Chattu, Pandya, Schroeder, Ray and Banach. This is an open-access article distributed under the terms of the Creative Commons Attribution License (CC BY). The use, distribution or reproduction in other forums is permitted, provided the original author(s) and the copyright owner(s) are credited and that the original publication in this journal is cited, in accordance with accepted academic practice. No use, distribution or reproduction is permitted which does not comply with these terms. 